\title{
Anti-Glucocorticoid Gene Therapy Reverses the Impairing Effects of Elevated Corticosterone on Spatial Memory, Hippocampal Neuronal Excitability, and Synaptic Plasticity
}

\author{
Theodore C. Dumas, ${ }^{1}$ Todd Gillette, ${ }^{1}$ Deveroux Ferguson, ${ }^{2}$ Kelly Hamilton, ${ }^{1}$ and Robert M. Sapolsky ${ }^{3}$ \\ ${ }^{1}$ Department of Molecular Neuroscience, Krasnow Institute for Advanced Study, George Mason University, Fairfax, Virginia 22030, ${ }^{2}$ Department of \\ Neuroscience, Mount Sinai School of Medicine, New York, New York 10029, and ${ }^{3}$ Department of Biological Sciences, Stanford University, Stanford, \\ California 94305
}

\begin{abstract}
Moderate release of the major stress hormones, glucocorticoids (GCs), improves hippocampal function and memory. In contrast, excessive or prolonged elevations produce impairments. Enzymatic degradation and reformation of GCs help to maintain optimal levels within target tissues, including the brain. We hypothesized that expressing a GC-degrading enzyme in hippocampal neurons would attenuate the negative impact of an excessive elevation in GC levels on synaptic physiology and spatial memory. We tested this by expressing 11- $\beta$ hydroxysteroid dehydrogenase (type II) in dentate gyrus granule cells during a $3 \mathrm{~d}$ GC treatment followed by examination of synaptic responses in hippocampal slices or spatial performance in the Morris water maze. In adrenalectomized rats with basal GC replacement, additional GC treatments for $3 \mathrm{~d}$ reduced synaptic strength and promoted the expression of long-term depression at medial perforant path synapses, increased granule cell and CA1 pyramidal cell excitability, and impaired spatial reference memory (without influencing learning). Expression of 11- $\beta$-hydroxysteroid dehydrogenase (type II), mostly in mature dentate gyrus granule cells, reversed the effects of high GC levels on granule cell and pyramidal cell excitability, perforant path synaptic plasticity, and spatial memory. These data demonstrate the ability of neuroprotective gene expression limited to a specific cell population to both locally and trans-synaptically offset neurophysiological disruptions produced by prolonged increases in circulating stress hormones. This report supplies the first physiological explanation for previously demonstrated cognitive sparing by anti-stress gene therapy approaches and lends additional insight into the hippocampal processes that are important for memory.
\end{abstract}

\section{Introduction}

Stress activates the hypothalamic-pituitary-adrenal (HPA) axis and increases circulating levels of the stress hormones glucocorticoids (GCs) (Kolber et al., 2008). An acute stress response provides physical and mental coping resources, but a prolonged response can have an array of adverse effects, including impairing memory functions dependent on the hippocampus (Kim et al., 2006). Thus, reducing the impact of prolonged elevations of GC levels is crucial to maintaining hippocampal integrity and normal memory function.

Learning and memory components of spatial navigation in rodents rely on hippocampal synaptic plasticity (Shapiro and Eichenbaum, 1999; Martin and Morris, 2002; Pittenger and Kandel, 2003). Stress or elevated GC levels have inverted-U effects on hippocampal synaptic function and spatial learning. For instance, a mild stressor or moderate GC levels enhances long-term potentiation (LTP) (Diamond et al., 1992; Pavlides et al., 1994)

Received Sept. 6, 2009; revised Dec. 1, 2009; accepted Dec. 10, 2009.

This work was supported by National Institutes of Health Grants R01NS32848, R01AG020633, and NIH5T32NSO7280 (R.M.S.), the Adler Foundation, and internal funds from the College of Science at George Mason University. We thank Dr. W. Ogle, Dr. D. Kaufer, and Z. Pincus for supplying the $11 \beta$ HSDII vector.

Correspondence should be addressed to Theodore C. Dumas, Department of Molecular Neuroscience, Krasnow Institute for Advanced Study, MS 2A1, George Mason University, Fairfax, VA 22030. E-mail: tdumas@gmu.edu. DOI:10.1523/JNEUROSCI.4402-09.2010

Copyright $\odot 2010$ the authors $\quad 0270-6474 / 10 / 301712-09 \$ 15.00 / 0$ and improves spatial memory (Pugh et al., 1997; Roozendaal and McGaugh, 1997; Liu et al., 1999; Akirav et al., 2004). In fact, moderate GC levels seem necessary for memory consolidation (Oitzl and de Kloet, 1992; Sandi et al., 1997; Conrad et al., 1999). Conversely, chronic stress or increased GC levels reduces LTP (Foy et al., 1987; Woolley et al., 1990; Diamond et al., 1992), promotes long-term depression (LTD) (Pavlides et al., 1995; Kim et al., 1996; Coussens et al., 1997; Xu et al., 1997; Yang et al., 2004), and impairs spatial memory (Luine et al., 1994; Oitzl et al., 1994; Diamond et al., 1996; Conrad et al., 1997; de Quervain et al., 1998). Thus, as circumstances shift from "stimulating" to "stressful," GCs shift from being beneficial to impairing (Kim and Yoon, 1998; de Kloet et al., 1999; Sauro et al., 2003). Inverted-U effects can be explained by high-affinity mineralocorticoid receptors (MRs) and lower-affinity glucocorticoid receptors (GRs) that act as positive and negative modulators, respectively (Reul and de Kloet, 1985; McEwen et al., 1986). However, the physiological disruptions produced in different subregions of the hippocampus during an excessive stress response are not well understood. Likewise, the specific physiological alterations produced by excessive GR activation that relate to impaired spatial cognition need additional investigation (Kim and Diamond, 2002).

The involvement of selective subsets of hippocampal neurons in specific aspects of spatial cognition can be tested by 
expressing factors that regulate GR activation in distinct neuronal subtypes during a period of excessive GC levels. Although $11-\beta$-hydroxysteroid dehydrogenase type $\mathrm{I}$ is native to the hippocampus, it works bidirectionally to both create and degrade corticosterone (CORT). The renal 11- $\beta$-hydroxysteroid dehydrogenase type II ( $11 \beta$ HSDII) acts only to degrade CORT (Seckl, 2004). In this study, we used a herpes amplicon viral vector to express $11 \beta$ HSDII preferentially in granule cells of the dentate gyrus of adult rats, in which MRs and GRs are highly expressed and colocalized (Han et al., 2005). We hypothesized that such expression would protect against the effects of excessive GC exposure and expose the impact of alterations in granule cell excitability and perforant path to dentate gyrus (PP-DG) synaptic transmission on spatial memory.

\section{Materials and Methods}

Vector construction. The plasmids containing the enhanced green fluorescent protein (eGFP) (GenBank accession number U55761) and the $11 \beta H S D I I$ genes (GenBank accession number U14631) have been described previously (Kaufer et al., 2004) and are variants of the standard bicistronic plasmid developed in our laboratory (Ho, 1994). For virus construction, E5 cells were transfected with p $\alpha 4$ eGFP (a plasmid carrying the eGFP gene under the control of the herpes simplex virus $\alpha 4$ promoter) or with $\mathrm{p} \alpha 411 \beta$ HSDIIp $\alpha 22 \mathrm{eGFP}$ (a bipromoter plasmid carrying $e G F P$ and $11 \beta H S D I I$ genes) (Fig. $1 A$ ) and then superinfected with d120 helper virus (DeLuca et al., 1985). After maximum infection, virus was released by sonication, partially purified (overlaid on $25 \%$ sucrose, $75 \mathrm{~kg}$ for $19 \mathrm{~h}$ at $4^{\circ} \mathrm{C}$ ) and resuspended in PBS. For simplicity, the vector with the p $\alpha 22$ eGFP plasmid is termed the eGFP vector, and the vector with the p $\alpha 411 \beta$ HSDIIp $\alpha 22 \mathrm{eGFP}$ plasmid is called the $11 \beta$ HSDII vector. Animals that express eGFP alone are referred to as controls.

Adrenalectomy surgery, vector delivery, and CORT injections. Male Sprague Dawley rats (250-350 g; Simonsen) were anesthetized with isoflurane (3-5\% vapor) and underwent bilateral adrenalectomy (ADX) and subcutaneous implantation of a CORT pellet (100 mg; 15\% CORT, $85 \%$ cholesterol). Drinking water was replaced with saline $(0.9 \%)$ after surgery. For animals in the behavioral experiments, the anesthetic was a $1 \mathrm{ml} / \mathrm{kg}$ injection of a ketamine, acepromazine, and xylazine mixture (10:2:1 ratio; 100,10 , and $100 \mathrm{mg} / \mathrm{ml}$ stock solutions, respectively).

Bilateral intracranial surgeries were performed $2-5 \mathrm{~d}$ after ADX surgery $(11 \beta$ HSDII and eGFP injections were performed on the same day for each cohort). Each animal was positioned in a stereotaxic device and had burr holes drilled in its skull to deliver eGFP (amplicon/helper ratio: $3.0 \times 10^{7}$ particles $/ \mathrm{ml}$ to $4.8 \times 10^{7}$ particles $/ \mathrm{ml}$ ) or $11 \beta$ HSDII vector $\left(1.2 \times 10^{7}\right.$ particles $/ \mathrm{ml}$ to $2.6 \times 10^{7}$ particles $\left./ \mathrm{ml}\right)$ into the dentate gyrus $(-3.6 \mathrm{~mm}$ anteroposterior and $+3.0 \mathrm{~mm}$ mediolateral from bregma, $-3.0 \mathrm{~mm}$ dorsoventral from dura; $0.5 \mu \mathrm{l} / \mathrm{min}$ for $3 \mathrm{~min}$ ). After surgery, animals received the first of three daily subcutaneous injections of either CORT $(10 \mathrm{mg} / \mathrm{kg})$ or vehicle (peanut oil) and a single intraperitoneal injection of bromodeoxyuridine (BrdU) (in sterile saline, $40 \mathrm{mg} / \mathrm{kg}$ ). When added to the basal CORT released by the subcutaneous pellet, these CORT injections produce chronically high levels of serum CORT $(\sim 28 \mathrm{mg} / \mathrm{dl})$ for $\sim 20 \mathrm{~h} / \mathrm{d}$ (Stein-Behrens et al., 1994). Three days of CORT injections were performed so that the animal healed adequately from intracranial surgery, and the vector still expressed at high levels at the time of testing (while at the same time ensuring that the CORT pellets maintained basal serum CORT levels throughout the experiment). All animal experimentation reported in this paper was conducted in accordance with the guidelines specified by the National Institutes of Health (NIH Guide for the Care and Use of Laboratory Animals), the Stanford Department of Veterinary Services and Care, and the Institutional Animal Care and Use Committee of George Mason University.

Hippocampal slice electrophysiology. Twenty-four hours after the final (third) CORT injection, hippocampal slices were prepared, and electrically evoked perforant path to dentate gyrus granule field responses were recorded (Dumas et al., 2000). Briefly, rats were anesthetized with isoflurane and decapitated, and the brain was placed into ice-cold oxygenated
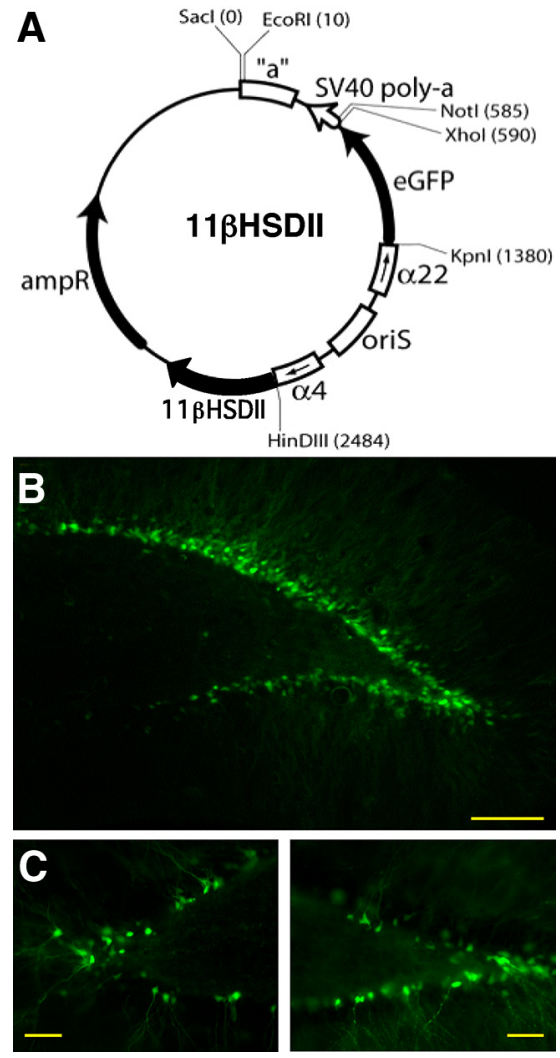

Figure 1. Plasmid map for $11 \beta \mathrm{HSD} \|$ and eGFP expression. $\boldsymbol{A}$, Diagram of the $11 \beta \mathrm{HSDII}$ plasmid showing the herpes oris origin of replication flanked by herpes $\alpha 4$ and $\alpha 22$ promoters. The eGFP gene is driven by the $\alpha 22$ promoter and ends with a simian virus poly(A) termination sequence (SV40). $11 \beta \mathrm{HSDII}$ was ligated to a cytomegalovirus poly(A) termination sequence (data not shown) and inserted at the HindIIII (2484) site just downstream of the $\alpha 4$ promoter. Also shown are the ampicillin resistance (ampR)-positive selection gene and packaging " $a$ " sequence that is necessary for inclusion of the plasmid into viral capsids. $\boldsymbol{B}$, eGFP expression from an animal in the MWM experiment that received the $11 \beta$ HSDII vector. Photos show eGFP-positive granule cells in a $33 \mu \mathrm{m}$ hippocampal section. Digital photo was taken at $10 \times$ magnification. Scale bar, $200 \mu \mathrm{m}$. C, Photos show eGFP-positive granule cells in $40 \mu \mathrm{m}$ sections (11ßHSDII, left; eGFP, right) from hippocampal slices used for electrophysiology. Digital photos were taken at $20 \times$ magnification. Scale bar, $100 \mu \mathrm{m}$.

(95\% $\mathrm{O}_{2}, 5 \% \mathrm{CO}_{2}$ ) artificial CSF (ACSF) (in mm: $124 \mathrm{NaCl}, 2 \mathrm{KCl}, 2$ $\mathrm{MgSO}_{4}, 2 \mathrm{CaCl}_{2}, 1.25 \mathrm{KH}_{2} \mathrm{PO}_{4}, 26 \mathrm{NaHCO}_{3}$, and 10 dextrose, $\mathrm{pH}$ 7.4). The hippocampus was dissected free and sliced parallel to the alvear fibers $(400-450 \mu \mathrm{m})$. A small penetration mark designating the cannula tract was apparent on each hippocampus. Slices close to, but not containing, the injection site were selected and transferred to an interface recording chamber (room temperature) to incubate for at least $2 \mathrm{~h}$ before recording.

To elicit PP-DG synaptic responses, a bipolar stimulating electrode (insulated platinum-iridium) was placed in contact with the middle molecular layer in the superior blade. A recording electrode (ACSF-filled glass pipette, $2-8 \mathrm{M} \Omega$ tip resistance) was positioned $\sim 1 \mathrm{~mm}$ from the stimulating electrode in the middle molecular layer to record the fiber potential (FP) and EPSP. A second recording electrode was placed in the hilus adjacent to the first recording electrode to record the population spike (PS). Responses were evoked at 25, 50, 100, and $200 \mu \mathrm{A}$. FP or PS amplitude was calculated as the voltage difference between cursors set at the initiation and negative peak of the response. EPSP slope was extracted from a $0.8 \mathrm{~ms}$ epoch at the initial downward deflection of the response (example waveforms are presented in Fig. 2). Response values were averaged at each stimulation level to construct input/output (I/O) curves. After collection of I/O curves, paired-pulse responses were elicited (25, 50,100 , and $200 \mathrm{~ms}$ interstimulus intervals) to create interstimulus interval (ISI) curves and test for changes in presynaptic function. For LTD 
experiments, stimulation intensity was adjusted to elicit a stationary 1 $\mathrm{mV}$ baseline EPSP for at least $15 \mathrm{~min}(0.033 \mathrm{~Hz}$ interpulse interval). LTD was induced with 900 pulses at $1 \mathrm{~Hz}$, and recording continued for $30 \mathrm{~min}$ at baseline frequency starting $15 \mathrm{~s}$ after LTD induction.

In some slices, Schaffer collateral (SC) field responses were recorded in the stratum pyramidale and stratum radiatum of area CA1 with a stimulating electrode positioned $\sim 1 \mathrm{~mm}$ away in the stratum radiatum near the CA2-CA1 border.

Spatial behavior in the Morris water maze. In a second group of subjects, spatial training ensued $24 \mathrm{~h}$ after the final CORT injection. Animals were trained in cohorts of four to six animals, and all groups were represented within each cohort. The maze consisted of a black circular pool ( $1.7 \mathrm{~m}$ diameter) filled with tap water $\left(24-27^{\circ} \mathrm{C}\right)$ to a level that covered a stationary black escape platform ( $15 \mathrm{~cm}$ diameter) by $1-2 \mathrm{~cm}$. The testing room $(2.7 \times 6.0 \mathrm{~m})$ had one black wall, one beige wall, and one checkered wall $(0.6 \times 0.6 \mathrm{~m}$ squares $)$ surrounding the pool. The fourth side was an open space containing the animal tracking equipment. Training was performed in six blocks of three trials, each with a 20-30 min interblock interval. The goal quadrant was varied across testing cohorts. Four starting locations, equally spaced from each other, were offset from the goal location by $45^{\circ}$. Starting location was chosen pseudorandomly from these positions for each trial. At the end of each trial, a 10 s latency on the platform was imposed. After the sixth training block (trials 16-18), a probe trial was performed in which the platform was removed and the rat was allowed to swim freely for $1 \mathrm{~min}$. With the platform replaced, three more trials were performed (trials 19-21). At $24 \mathrm{~h}$ after training, a second probe trial was performed. For each training trial, latency to escape, path length to escape, and mean swim speed were calculated. For each probe trial, the amount of time searching in each quadrant (dwell time) was recorded.

Histology. Immediately after electrophysiological recording, hippocampal slices were fixed in 3\% paraformaldehyde (PF) (dissolved in PBS) for 10-20 min and refrigerated $\left(4^{\circ} \mathrm{C}\right)$ in PBS overnight. Slices were then embedded in gelatin (300 bloom, 14\%) and postfixed in PF (24-48 h). Forty micrometer sections were cut with a vibratome and mounted on gelatin-coated slides.

Immediately after the $24 \mathrm{~h}$ probe trial, rats were perfused with $\sim 60-80 \mathrm{ml}$ of heparinized saline (2000 U/L; Acros Organics), followed by a similar volume of $3 \%$ PF. Brains were placed in $30 \%$ sucrose in PF for $48-96 \mathrm{~h}$, after which they were frozen to -17 to $-19^{\circ} \mathrm{C}$ and cut in the coronal plane with a cryostat. Brains from four animals were hemisected, embedded in gelatin, and sectioned with a vibratome ( $33 \mu \mathrm{m}$, all sections containing dorsal hippocampus were kept). For cryostat-cut sections, beginning with the first section that showed a distinct DG granule cell layer, every third section ( $33 \mu \mathrm{m}$ thickness) was saved and mounted on a slide until the dorsal and ventral hippocampus were no longer viewed as distinct structures.

Immunohistochemistry was performed on vibratome-cut sections from animals trained in the Morris water maze (MWM). Sections were first washed in Tris-buffered saline (TBS), and those designated for BrdU labeling were incubated in $2 \mathrm{~N} \mathrm{HCl}$ for $10 \mathrm{~min}$. All tissue was made permeable in $1 \%$ Triton X-100 (in TBS, $20 \mathrm{~min}$ ) and blocked with $10 \%$ normal goat serum (in TBS, $20 \mathrm{~min}$ ), followed by overnight incubation in primary antibody [anti-BrdU (Millipore Corporation) or antidoublecortin (DCX) (Santa Cruz Biotechnology)] at a 1:200 dilution in TBS plus Triton X-100 (0.3\%) and normal goat serum (3\%). Sections were washed three times in TBS and incubated in secondary antibody [tetramethylrhodamine isothiocyanate-conjugated anti-goat or cyanine 3 (Cy3)-conjugated anti-mouse; Millipore Corporation] at a 1:200 dilution in TBS plus Triton X-100 (0.3\%) and normal goat serum (3\%). Granule cells were visualized with epifluorescence at 475/505 nm (peak excitation/peak emission) for eGFP and 555/565 nm for Cy3 (Olympus BX51WIF). Exposure times and lamp intensity values were identical when imaging sections treated with and without primary antibody.

Parameter extraction and statistics. Recording and parameter extraction were performed blind to experimental condition. For each animal, EPSP slope values were averaged at each level of the I/O curve, and these values were averaged by treatment group. I/O curves and ISI curves were first analyzed by three-way repeated-measures ANOVA, and significant tests were followed by two-way repeated-measures ANOVAs. Parameters extracted from CA1 recordings were compared by two-way ANOVA and unpaired $t$ tests (Bonferroni's-corrected).

For LTD analysis, response values from the final $10 \mathrm{~min}$ of baseline stimulation were averaged, and all responses were divided by this mean to produce normalized proportion of baseline values $(\times 100$ for percentage of baseline). LTD values were compared between groups by two-way ANOVA (hormone $\times$ vector). Within each group, induction of LTD was determined by a paired $t$ test comparing the mean EPSP value at 25-30 min after $1 \mathrm{~Hz}$ stimulation to the baseline mean.

For analysis of spatial learning in the MWM, escape latencies, dwell times, and mean swim speeds for each animal were averaged by training block. Block means were averaged by treatment group and compared by three-way repeated-measures ANOVA (hormone $\times$ vector $\times$ block). To assess memory performance in the immediate and $24 \mathrm{~h}$ probe trials, dwell times for each quadrant were averaged by treatment group. $\chi^{2}$ tests were performed to determine whether any quadrant bias was displayed among the subjects in each group. A two-way ANOVA was used to compare dwell time in goal quadrant between groups. One-group $t$ tests were performed to compare dwell time in the goal quadrant to chance (15 s) during the immediate and $24 \mathrm{~h}$ probe trials.

Error bars in all figures indicate SEM.

\section{Results}

Delivery of $11 \beta$ HSDII amplicons to the dentate gyrus produces dense and selective infection of granule cells

Under fluorescent microscopy, eGFP-positive cells in infected hippocampi were observed in the superior and inferior blades of the DG, with $20-30 \%$ of total granule cells infected (Fig. $1 B$ ). No eGFP-positive neurons were observed in area CA1, and only a handful of non-granule eGFP-positive cells were seen elsewhere across all animals and slices. Higher-magnification images of sections from slices used for electrophysiology show distinct fluorescent granule cells and dendrites (Fig. 1C). Fluorescent granule cells were typically observed at both faces of the 400-mm-thick slice and at three slices away from the injection site, amounting to $\sim 3 \mathrm{~mm}$ total spread along the longitudinal axis of the hippocampus; this is in agreement with previous studies using this delivery system and a colorimetric (McLaughlin et al., 2000; Dumas et al., 2004) or fluorescent (Nicholas et al., 2006; Ferguson and Sapolsky, 2008) reporter.

\section{Expression of $11 \beta \mathrm{HSDII}$ reverses the increase in granule cell excitability, but not the depression of synaptic transmission, produced by high CORT}

Electrically evoked medial PP-DG synaptic field responses were recorded simultaneously in the molecular and granule cell layers of the superior blade at varying stimulation intensities to produce I/O curves (Fig. $2 A$ ). At the $25 \mu \mathrm{A}$ stimulation intensity, EPSP values in numerous slices were below or near threshold, making calculations unreliable; thus, this stimulation level was omitted from additional analyses. A three-way repeated-measures ANOVA for the effects of CORT level, vector type, and stimulation intensities on the FP amplitudes showed only a repeatedmeasures effect $\left(F_{(3,287)}=11.47, p<0.0001\right)$, indicating that the $\mathrm{FP}$ responses increased across stimulation intensities but were not affected by hormone or vector conditions. In contrast, the EPSP slope was decreased in slices from high-CORT animals compared with slices from low-CORT animals $\left(F_{(1,287)}=8.93\right.$, $p<0.005$ ), but there was no effect of vector, reflecting a synaptic depression produced by high CORT that was not altered by vector delivery. Likewise, the ratio of the EPSP to FP was reduced by high CORT $\left(F_{(1,287)}=19.09, p<0.0001\right)$ but not altered by vector, further supporting a CORT-induced synaptic depression 


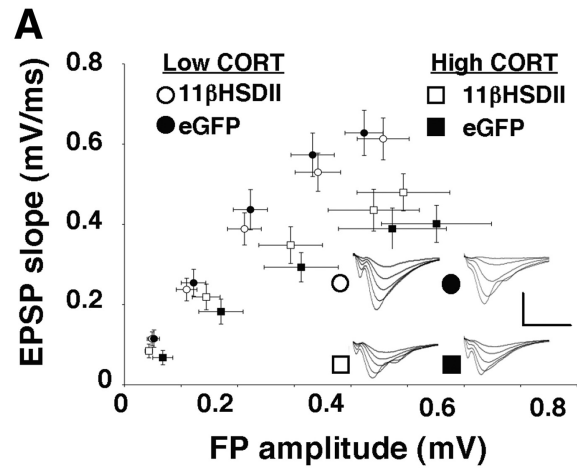

B

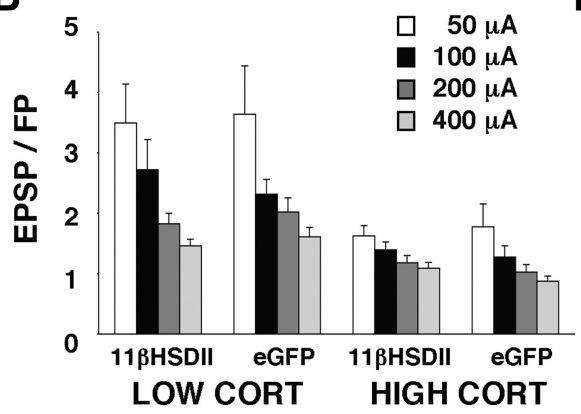

C
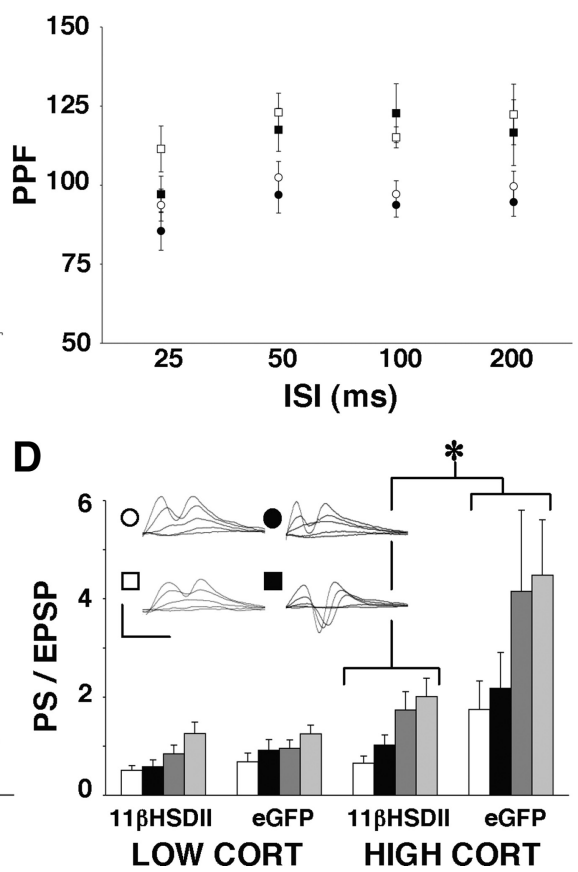

Figure 2. Effects of CORT and $11 \beta \mathrm{HSDII}$ expression on PP-DG baseline synaptic strength and granule cell excitability. A, I/0 curve shows mean EPSP slope values plotted against mean FP amplitude values for all groups (low CORT/11 $\beta$ HSDII, $n=19$; low CORT/eGFP, $n=19$; high CORT/11 $\beta$ HSDII, $n=16$; high CORT/eGFP, $n=18$ ). Inset shows averaged waveforms of responses recorded in the middle molecular layer at all stimulation intensities ( 5 sweeps per level). Calibration: $1 \mathrm{mV}, 10 \mathrm{~ms}$. $\boldsymbol{B}$, Bar graph shows mean EPSP to FP ratios plotted by stimulation intensity for each condition. $\boldsymbol{C}$, ISI curve shows mean percentage PPF values plotted against ISI values. D, Bar graph shows PS/EPSP ratio across stimulation intensities for each group. ${ }^{*} p<0.025$ by ANOVA. Inset shows averaged waveforms ( 10 sweeps each) of responses recorded in the granule cell layer at all stimulation intensities. Brackets and asterisk denote significant post hoc two-way repeatedmeasures ANOVA.

that was not reversed by expression of $11 \beta$ HSDII or GFP (Fig. $2 B$ ).

It is possible that the $11 \beta \mathrm{HSDII}$ vector did not protect against the reduction in baseline synaptic strength produced by high CORT because it was expressed postsynaptically and the source of the synaptic depression was presynaptic in origin. To test for presynaptic involvement, we delivered paired stimulation pulses and examined presynaptically mediated short-term plasticity. Whereas Schaffer collateral, mossy fiber, and lateral perforant path synapses express paired-pulse facilitation (PPF), medial perforant path synapses typically do not and even display slight paired-pulse depression (Kahle and Cotman, 1993; DiScenna and Teyler, 1994). In agreement, under low-CORT conditions, slices that expressed $11 \beta \mathrm{HSDII}$ or controls showed no PPF across ISI curves (Fig. 2C), and paired-pulse responses were not different between these two groups (two-way repeated-measures ANOVA), indicating no effect of $11 \beta$ HSDII on presynaptic function. The PPF ratio observed in slices from high-CORT animals was significantly greater than that observed for slices from low-CORT animals (threeway repeated-measures ANOVA, $F_{(1,127)}=$ 14.82, $p<0.001)$. An elevation in PPF is normally indicative of a decrease in transmitter release (Creager et al., 1980; Foster and McNaughton, 1991). Thus, the findings strongly suggest that the synaptic depression produced by elevated CORT was attributable at least in part as a reduction in presynaptic transmitter release, which may explain the inability of postsynaptic $11 \beta \mathrm{HSDII}$ expression to reverse the depression of transmission.

Recording in the granule cell layer allowed for observation of changes in the PS, a measure of granule cell excitability. The PS amplitude to EPSP slope ratio was calculated for each data point and averaged at each stimulus level for each experimental group (Fig. 2D). A three-way ANOVA revealed effects of hormone $\left(F_{(1,219)}=12.85, p<0.001\right)$, vector $\left(F_{(1,211)}=5.96, p<0.02\right)$, and a hormone $\times$ vector interaction $\left(F_{(1,287)}=4.23, p<0.05\right)$. Compared with the low-CORT animals, PS/EPSP values for the high-CORT/eGFP $\left(F_{(1,191)}=18.97, p<0.0001\right)$ and highCORT/11 $\beta$ HSDII $\left(F_{(1,191)}=4.31, p<0.05\right)$ animals were increased, reflecting a CORT-induced increase in granule cell excitability. The PS/EPSP values for the high-CORT/11 $\beta$ HSDII animals were significantly reduced when compared with the high-CORT/eGFP $\left(F_{(1,143)}=5.50, p<0.025\right.$, denoted by the asterisk), indicating protection against the CORT-induced increase in granule cell excitability by expression of $11 \beta$ HSDII.

The increased susceptibility to LTD induction produced by high CORT is blocked by expression of $11 \beta$ HSDII

For plasticity experiments, baseline responses were set to $1 \mathrm{mV}$ in amplitude, approximately one-third to one-half of the maximum EPSP amplitude for each group. The baseline EPSP slope was not different between groups (in $\mathrm{mV} / \mathrm{ms}$ : low CORT/11 $\beta$ HSDII, $-0.35 \pm 0.02, n=10$; low CORT/eGFP, $-0.29 \pm 0.02, n=10$;
Figure 3. Effects of CORT and $11 \beta \mathrm{HSD}$ Il expression on LTD. $\boldsymbol{A}$, Timelines for mean normalized responses before and after LTD induction in low-CORT animals. Inset shows overlaid aver aged waveforms representing responses before and after LTD induction (baseline, 20 sweeps; after induction, 10 sweeps). Calibration: $1 \mathrm{mV}, 10 \mathrm{~ms}$. $\boldsymbol{B}$, Timelines for mean normalized responses before and after LTD induction in high-CORT animals. Inset shows overlaid averaged waveforms representing responses before and after LTD induction. ${ }^{*} p<0.0001$, significant difference from baseline at $20-25 \mathrm{~min}$ after termination of induction. 
Table 1. Parameters extracted from $1 \mathrm{mV}$ EPSPs in area CA1

\begin{tabular}{|c|c|c|c|c|}
\hline \multirow[b]{2}{*}{ Parameter } & \multicolumn{2}{|l|}{ Low CORT } & \multicolumn{2}{|l|}{ High CORT } \\
\hline & $11 \beta \mathrm{HSDII}$ & eGFP & $11 \beta \mathrm{HSDII}$ & eGFP \\
\hline Number of slices & 12 & 12 & 12 & 12 \\
\hline Stimulation intensity $(\mu \mathrm{A})$ & $47 \pm 10$ & $74 \pm 32$ & $84 \pm 13$ & $96 \pm 19$ \\
\hline EPSP slope (mV/ms) & $-0.22 \pm 0.02$ & $-0.22 \pm 0.04$ & $-0.23 \pm 0.02$ & $-0.21 \pm 0.02$ \\
\hline PPF (\% of baseline) & $127 \pm 10^{a}$ & $126 \pm 5$ & $127 \pm 3^{a}$ & $117 \pm 4^{a}$ \\
\hline PS amplitude (mV) & $0.67 \pm 0.13$ & $0.65 \pm 0.16$ & $0.51 \pm 0.10^{* *, * * *}$ & $1.37 \pm 0.18^{*}$ \\
\hline
\end{tabular}

${ }^{*} p<0.05$, difference from combined low-CORT groups; ${ }^{* *} p<0.01$, difference from combined Low-CORT groups; ${ }^{* * *} p<0.005$, difference from high-CORT/eGFP group.

${ }^{a} n=8$ for PPF, low CORT/11 $\beta$ HSDII, high CORT/11 $\beta$ HSDII, and high CORT/eGFP.

high CORT/11bHSDII, $0.26 \pm 0.03, n=9$; high CORT/eGFP, $0.29 \pm 0.02, n=10$ ). However, a two-way ANOVA (hormone $\times$ vector) comparing mean normalized EPSP slope values at 25-30 min after LTD induction was significant $\left(F_{(3,38)}=6.91, p<\right.$ $0.005)$. When comparing the EPSP slope during the last $10 \mathrm{~min}$ of baseline and 25-30 min after LTD induction, no difference was observed for the low-CORT/11 $\beta$ HSDII (percentage of baseline, $101 \pm 5 \%$ ) or low-CORT/eGFP (percentage of baseline, $95 \pm$ $6 \%$ ) slices. Thus, as shown previously in slices from naive adult rats, LTD was not induced under basal CORT conditions (Dudek and Bear, 1993; Wagner and Alger, 1995) (Fig. 3A). In agreement with Kim et al. (1996), in high-CORT control animals, $1 \mathrm{~Hz}$ stimulation produced LTD (percentage of baseline, $77 \pm 7 \%$; paired $t$ test, $\left.t_{(18)}=2.1, p<0.0001\right)$. In contrast, when $11 \beta \mathrm{HSDII}$ was expressed in slices from high-CORT animals, no LTD was observed (percentage of baseline, $100 \pm 5 \%$ ), indicating protection against the increased susceptibility to LTD induction produced by high CORT.

\section{High CORT produces an increase in CA1 pyramidal cell excitability that is offset by expression of $11 \beta$ HSDII in DG granule cells}

In some slices, simultaneous recordings were made in the stratum pyramidale and stratum radiatum in area CA1 during stimulation of Schaffer collateral axons. Stimulation was set to evoke a 1 $\mathrm{mV}$ EPSP in the stratum radiatum. The stimulation intensities, EPSP slopes, and PPF (50 ms ISI) for $1 \mathrm{mV}$ EPSPs were not different between groups (Table 1), indicating that CORT condition or vector expression in DG granule cells did not affect baseline synaptic function at Schaffer collateral synapses.

CA1 pyramidal cell excitability was assessed by measuring the amplitude of the PS recorded in the stratum pyramidale at stimulation intensities that elicited $1 \mathrm{mV}$ EPSPs in the stratum radiatum. Two-way ANOVAs revealed no differences in stimulation intensity to achieve a $1 \mathrm{mV}$ EPSP or the EPSP slope at a $1 \mathrm{mV}$ amplitude (Table 1). However, for PS analyses, there was a main effect of vector $\left(F_{(3,47)}=8.05, p<0.01\right)$ and a hormone $\times$ vector interaction $\left(F_{(3,47)}=9.09, p<0.005\right)$. The effect of hormone likely did not reach significance $(p<0.07)$ because of the reduction in the PS amplitude in the high-CORT/11 $\beta$ HSDII groups below the PS amplitude of the low-CORT groups. The PS amplitudes were larger in the high-CORT/eGFP than in the combined low-CORT slices $\left(t_{(24)}=2.1, p<0.05\right)$, indicating that high CORT increased CA1 pyramidal cell excitability. In contrast, the high-CORT/11 $\beta$ HSDII animals displayed PS amplitudes that were of reduced magnitude when compared with either the lowCORT animals $\left(t_{(26)}=2.1, p<0.005\right)$ or the high-CORT/eGFP animals $\left(t_{(20)}=2.1, p<0.0001\right)$. Combined, the data show that $11 \beta$ HSDII expression in DG granule cells, two synapses away, prevented the CORT-induced increase in CA1 pyramidal cell excitability.

\section{Expression of $11 \beta \mathrm{HSDII}$ restores spatial memory in the MWM}

We used the MWM (Morris, 1981) to assess the effects of high CORT and $11 \beta$ HSDII on hippocampal-dependent spatial learning. Three days after vector delivery and the onset of CORT injections, animals were trained to find a hidden platform in a circular pool. As a whole, all groups displayed learning by reducing their escape latencies across training blocks $\left(F_{(5,190)}=20.40\right.$, $p<0.001$, does not include training data taken after the immediate probe trial; low CORT/11 $\beta$ HSDII, $n=9$; low CORT/eGFP, $n=10$; high CORT/11 $\beta$ HSDII, $n=11$; high CORT/eGFP, $n=$ $8)$. There was no difference between groups in the average latency (Fig. 4A) or mean path length (data not shown) to find the platform across learning curves. Thus, granule cell infection and $3 \mathrm{~d}$ of systemically elevated CORT do not produce measurable changes in sensorimotor abilities, working memory, or spatial navigation.

Search strategy probes for the platform location were performed both on the day of training (Fig. $4 B$ ) and $24 \mathrm{~h}$ later (Fig. $4 C) \cdot \chi^{2}$ tests were performed to detect spatial biases. On the same day of training, all groups showed a quadrant bias (low CORT/ $11 \beta$ HSDII, $\chi_{(3)}=13.67, p<0.01$; low CORT/eGFP, $\chi_{(3)}=11.60$, $p<0.01$; high CORT/11 $\beta$ HSDII, $\chi_{(3)}=9.73, p<0.02$; high CORT/eGFP, $\left.\chi_{(3)}=12.00, p<0.01\right)$. As well, all groups spent more time in the goal quadrant than would be predicted by chance $(15 \mathrm{~s}, 25 \%)$ (low-CORT/11 $\beta$ HSDII, $t_{(6)}=3.40, p<0.01$; low-CORT/eGFP, $t_{(9)}=7.60, p<0.0001$; high-CORT/ $11 \beta \mathrm{HSDII}_{(9)}=5.20, p<0.005$; high-CORT/eGFP, $t_{(6)}=$ $3.99, p<0.01$ ), and a two-way ANOVA (hormone $\times$ vector) for dwell time in the goal quadrant showed no main effects or interaction, indicating that all groups used a spatial search strategy on the same day of training.

For the $24 \mathrm{~h}$ probe, $\chi^{2}$ tests were significant for the lowCORT/eGFP group $\left(\chi_{(3)}=11.60, p<0.01\right)$ and the high-CORT/ $11 \beta$ HSDII group $\left(\chi_{(3)}=9.23, p<0.05\right)$, with a strong trend for significance for the low-CORT/11 $\beta$ HSDII group $\left(\chi_{(3)}=6.56\right.$, $p<0.088$ ), suggesting unequal dwell times in the four quadrants for all but the high-CORT/eGFP animals. A two-way ANOVA for dwell time in the goal quadrant produced an effect of vector $\left(F_{(1,35)}=4.29, p<0.05\right)$ but not hormone and no interaction. Low-CORT animals spent more time in the goal quadrant than would be predicted by chance (low-CORT/11 $\beta$ HSDII, $t_{(8)}=$ 3.30, $p<0.05$; low-CORT/eGFP, $\left.t_{(9)}=3.17, p<0.05\right)$, thus displaying memory for the goal quadrant. Animals that received high CORT and expressed eGFP did not show a bias for the goal quadrant, indicating that high CORT impaired spatial memory. However, similar to the low-CORT groups, animals that received high CORT and expressed $11 \beta$ HSDII spent more time in the goal quadrant $\left(t_{(10)}=2.50, p=0.05\right)$, supporting the idea that $11 \beta$ HSDII expression in DG granule cells reduced the negative effects of high CORT on spatial memory. 

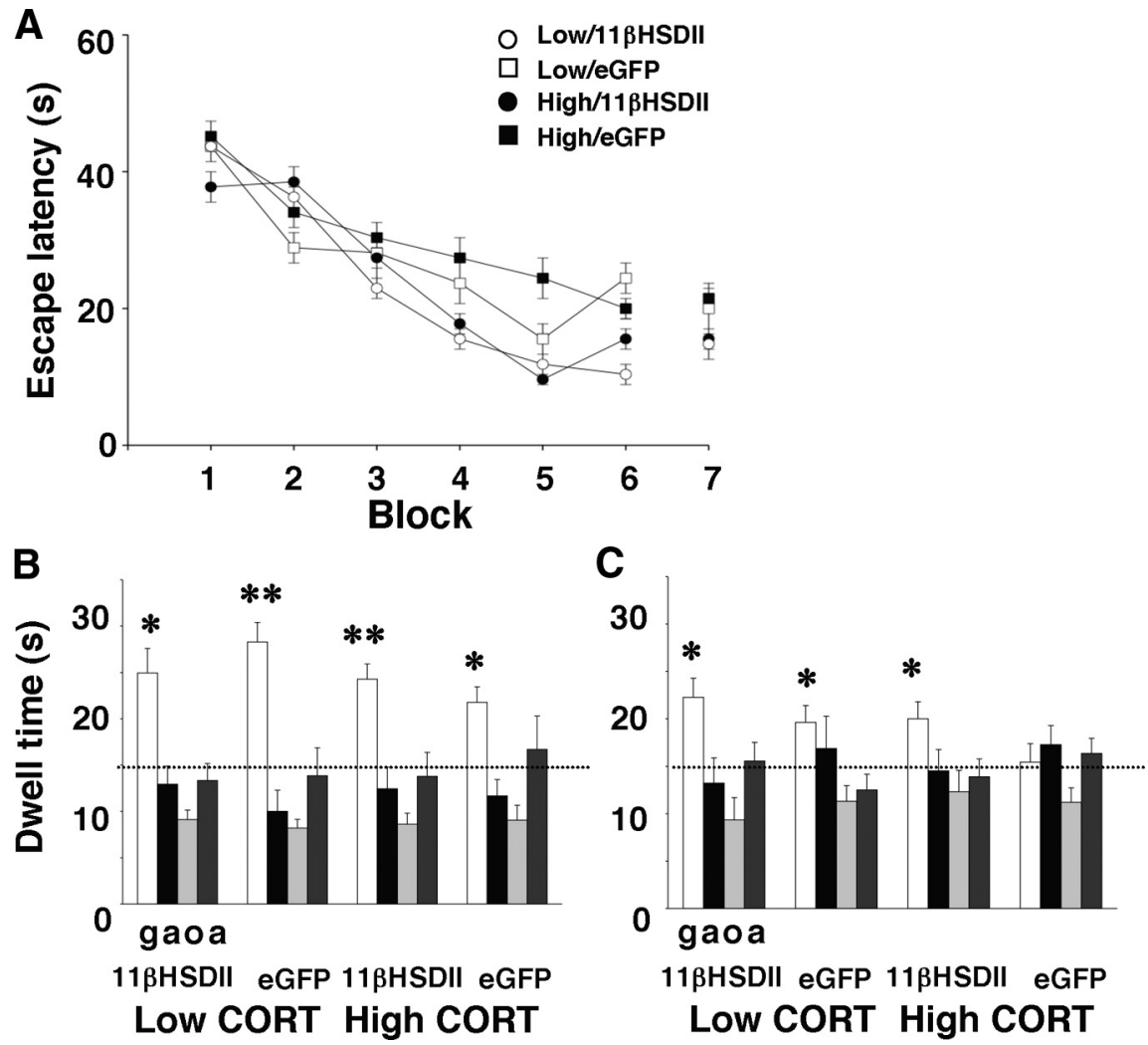

Figure 4. Learning curves and probe trial dwell times in the MWM. $\boldsymbol{A}$, Learning curves show mean escape latencies across training block for each group. $\boldsymbol{B}$, Histograms show mean dwell time for each quadrant ( $g$, goal; a, adjacent; 0, opposite) during the immediate probe trial. ${ }^{*} p<0.05,{ }^{* *} p<0.005$, significant difference from chance (15 s) in post hoc one-group $t$ tests. C, Histograms show mean dwell time for each quadrant during the $24 \mathrm{~h}$ probe trial.

\begin{abstract}
Amplicons infect both mature and immature granule cells as shown by colocalization of eGFP with doublecortin

It is possible that physiological and behavioral sparing was mediated via an effect on neurogenesis or on differential effects of CORT or viral infection on neurons of different maturational states. Vibratome-cut hemisections from four animals trained in the MWM were subjected to immunohistochemistry for the neurogenesis marker BrdU and the developmentally restricted neurofilament protein DCX. Nearly all sections containing eGFP-positive granule cells also showed positive labeling for BrdU and DCX. There was no obvious difference in the number of BrdU- or DCX-positive cells across animals. There was no evidence of colocalization of eGFP with BrdU (Fig. 5A1,A2) nor, in most instances, with DCX (Fig. 5B1,B2). However, there were cases in both low-CORT and high-CORT animals in which eGFP was colocalized with DCX (Fig. $5 B 1 a, B 1 b$, respectively). These data indicate that, although newly generated cells in the dentate gyrus are not subject to amplicon infection, both mature and immature neurons can be infected but with no preferential bias toward infecting the latter.
\end{abstract}

\section{Discussion}

Although pharmacological elevations in serum CORT are not equivalent to behavioral stress, the selective effect of CORT on spatial memory corroborates previous research showing that $3 \mathrm{~d}$ of rotation stress, $7 \mathrm{~d}$ of restraint stress, or $21 \mathrm{~d}$ of CORT treatments impairs reference memory, leaving learning and working memory intact. Our finding that high CORT elicits LTD also mimics the facilitating effect of behavioral stress on hippocampal LTD induction (Pavlides et al., 1995; Kim et al., 1996; Coussens et al., 1997; Xu et al., 1997; Yang et al., 2004). The current experiments further demonstrate that a $3 \mathrm{~d}$ CORT treatment increases neuronal excitability and produces a lasting depression of baseline PP-DG synaptic transmission that is, in part, presynaptic in origin. The effects of elevated CORT on hippocampal synaptic transmission and spatial reference memory may be indirect, involving a change in sleep patterns or other systemic influence of HPA axis manipulation. Our findings implicate the actions of CORT in DG granule cells because the alterations in granule cell excitability, PP-DG synaptic LTD, and spatial memory were all reversed by $11 \beta$ HSDII expression specifically within these neurons.

Approximately $20-30 \%$ of the DG granule cell population was infected, with a bias toward mature neurons, which corroborates more detailed previous analyses (McLaughlin et al., 2000). No pyramidal cell infection was observed. Thus, many unprotected neurons in the animals that did not express $11 \beta$ HSDII could have contributed to the inability of $11 \beta$ HSDII vector delivery to fully preserve spatial memory. Because $11 \beta$ HSDII expression reduced the impact of high CORT on granule cell excitability, LTD induction, and spatial memory, it appears that plasticity at DG inputs or DG excitability could regulate spatial memory. Although changes in memory consolidation cannot be ruled out, sparing effects on recall are supported by the demonstration that expression of a transdominant GR in DG granule cells can prevent the disruption of long-term spatial memory produced by a CORT injection delivered $30 \mathrm{~min}$ before the memory test (Ferguson and Sapolsky, 2008).

Cognitive disruption and dendritic retraction in area CA3 pyramidal cells result from chronic stress or repeated CORT treatment (Woolley et al., 1990; Arbel et al., 1994; Conrad et al., 1996). It is unlikely that dendritic atrophy was created by our $3 \mathrm{~d}$ CORT treatment because it is not produced by 2 weeks of stress (Magariños and McEwen, 1995) and the chronic stress paradigms that produce dendritic atrophy also impair learning (Arbel et al., 1994; Conrad et al., 1996), which was not apparent in this study. More recent work showed that reducing CORT levels during memory assessment prevents chronic stress-induced impairments in spatial memory (Wright et al., 2006), suggesting that the cognitive effect is more closely related to CORT level than to dendritic atrophy.

A persistent alteration in synaptic plasticity $24 \mathrm{~h}$ after the final CORT injection corroborates previous findings in area CA1 and the DG after the termination of a chronic stressor (Pavlides et al., 1993; Alfarez et al., 2003). Both PP-DG and SC-CA1 synapses are more resistant to LTP and more susceptible to LTD induction after chronic stress (Kim et al., 1996; Coussens et al., 1997; Xu et al., 1997). Regional differences in CORT sensitivity are supported by a reduction in baseline synaptic strength at PP-DG synapses not observed at SC-CA1 synapses (current results) and a greater stress-induced shift in metaplasticity at PP-DG synapses (Abraham 
and Tate, 1997). Whether the effects of CORT on synaptic transmission and plasticity are an indirect result of a more global cellular alteration such as a change in neuronal excitability (Joëls and de Kloet, 1992), calcium channels (Joëls et al., 2003), or cellular metabolism (Takahashi et al., 2002) is an interesting question for follow-up research.

It is unclear whether the increase in neuronal excitability we observed reflects the state of the system during CORT treatment or is a rebound effect. Previous studies have shown suppressed hippocampal activity with an acute CORT injection (Pfaff et al., 1971) or an early increase in excitability followed by a suppression (Joëls and de Kloet, 1990). Given the same time course, modest increases in CORT can suppress excitability, whereas larger increases augment excitability because of differences in MR and GR occupation (Joëls et al., 1994). Thus, when interpreting effects of stress or CORT on neuronal excitability, it is clearly important to consider both level and time course of the treatment as well as the time of assay relative to treatment (during or after). Interesting follow-up experiments might include a CORT injection on the day of assay or multiple assessments at differing time points after the final CORT delivery.

The ability of $11 \beta$ HSDII expression in DG granule cells to maintain low excitability levels in area CA1 during high CORT was unexpected and was not the result of extra-DG infection. The most harmonious explanation is that, even in the slice preparation, activity levels in the DG (or entorhinal cortex) affect activity levels in downstream regions. Resulting caveats are that the impact of CORT in CA1 is not entirely attributable to direct effects in this region, and the $11 \beta$ HSDIIdependent reduction in CA 1 excitability is likely secondary to the reduction in DG excitability.

The observation that $3 \mathrm{~d}$ of elevated CORT reduced baseline PP-DG synaptic transmission is comparable with the time course of synaptic depression that occurs at this relay after ADX without CORT replacement (Stienstra et al., 1998), consistent with the inverted-U effects of CORT in the hippocampus. The highCORT-induced synaptic depression was not reversed by expression of $11 \beta$ HSDII, possibly as a result of a difference in the site of the impairment and $11 \beta$ HSDII expression (presynaptically and postsynaptically, respectively). Alternatively, it has been shown that CORT mediates a suppression of PP-DG synaptic transmission via effects in the basolateral amygdala (Vouimba et al., 2007 ), which might not be offset by $11 \beta$ HSDII expression in DG granule cells. Interestingly, the reduction in baseline synaptic function did not impact spatial learning and was not related to the improvement in spatial memory in animals expressing $11 \beta$ HSDII.

Occupation of MRs, perhaps with a very low level of GR occupancy, is necessary to maintain synaptic function (Stienstra et al.,

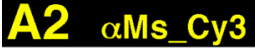

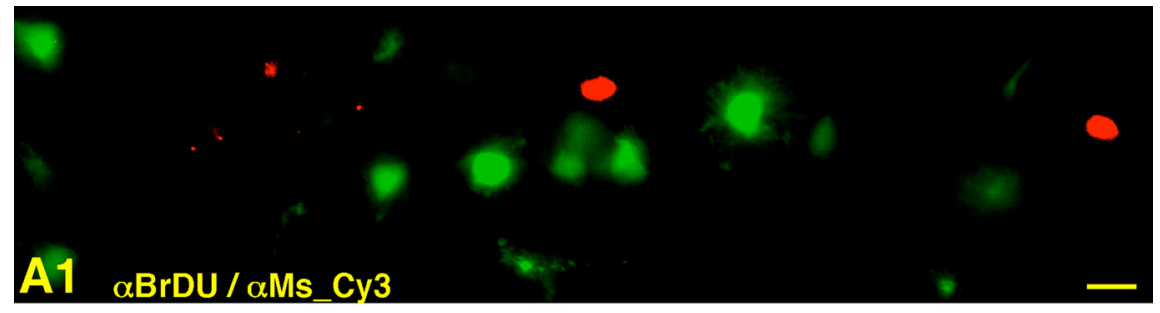
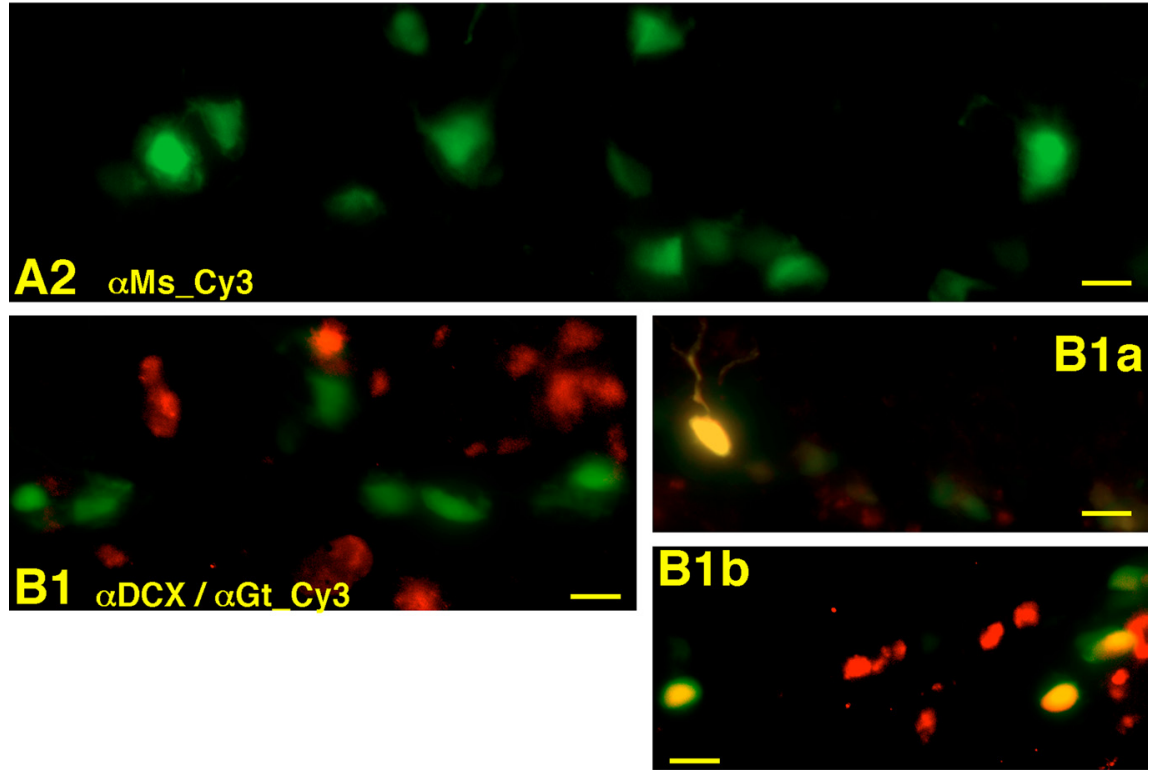

Figure 5. Fluorescent images showing eGFP in infected dentate gyrus granule cells and immunohistochemistry results for BrdU and $D C X$. All images are of the granule cell layer in the superior blade with dendrites pointing upward (40X magnification) A1, Infected neurons (green) do not colocalize with BrdU (red). A2, There is no red fluorescence in sections not treated with tion between eGFP and DCX could be found in low-CORT (B1a) and high-CORT (B1b) animals. B2, There is no red fluorescence in sections not treated with anti-DCX primary antibody. $\alpha \mathrm{Ms}$, Anti-mouse; $\alpha \mathrm{Gt}$, anti-goat.

1998 ) and is clearly demonstrated by recent experiments that use MR-specific agonists and antagonists (Avital et al., 2006). If $11 \beta$ HSDII were to completely degrade CORT, one might expect a far left shift on the inverted-U curve in which MRs are not occupied by CORT, resulting in no benefits for synaptic transmission and memory. This was not observed. It is possible that $11 \beta$ HSDII does not completely degrade CORT or that cortisone has sufficient efficacy at MRs. Combined with the higher affinity of MRs for CORT, it appears that $11 \beta$ HSDII expression preferentially reduces occupancy of GRs. This idea is supported by a previous report from our laboratory showing that $11 \beta \mathrm{HSDII}$ is equally efficacious as a virally expressed transdominant GR in reducing the endangering effects of CORT on excitotoxicity in vitro and in vivo (Kaufer et al., 2004).

New neurons are continually generated in the DG, many of which mature to become incorporated into the hippocampal circuit (Li et al., 2009). The rate of neurogenesis in the DG is reduced by chronic stress (Gould et al., 1991; Tanapat et al., 1998; Malberg and Duman, 2003), leaving open the possibility that CORT im- 
pacted DG physiology and MWM performance in our study via a reduction in neurogenesis. It seems unlikely that our $3 \mathrm{~d}$ CORT treatment would reduce proliferation enough to produce observable effects on memory or on excitability and synaptic transmission measured at the population level (Kempermann, 2002), although it should be noted that, at any given time, neurons of all developmental stages are present in the DG. As such, there could be maturation-dependent effects of CORT and/or viral infection. Newborn cells that do not express GR or MR for the first $3 \mathrm{~d}$ are those that are presumed to survive to become fully functional neurons (Garcia et al., 2004). Immature neurons express both MR and GR and, as shown here, can be infected by herpes amplicons. Mature and immature neurons contribute differentially to perforant path synaptic plasticity (Wang et al., 2000; Snyder et al., 2001; Schmidt-Hieber et al., 2004) and to memory function (Snyder et al., 2005; Saxe et al., 2007), so it is important to consider developmental state. Nevertheless, the physiology and memory restoring effects of $11 \beta$ HSDII in this study appear to be attributable to its expression in mature DG granule cells because that was the predominant site of eGFP expression.

The current findings demonstrate that various negative impacts on hippocampal function resulting from prolonged elevations in circulating stress hormones are offset by neuroprotective gene expression in a subset of DG granule cells. Effects include normalized PP-DG synaptic plasticity and both local and transsynaptic neuronal excitability. The current physiological results may explain reports of cognitive sparing produced by similar anti-stress gene therapy approaches. Relationships between the physiological and behavioral changes under high-CORT conditions and with $11 \beta$ HSDII expression allow for a greater understanding of the specific neuronal mechanisms that support learning and memory.

\section{References}

Abraham WC, Tate WP (1997) Metaplasticity: a new vista across the field of synaptic plasticity. Prog Neurobiol 52:303-323.

Akirav I, Kozenicky M, Tal D, Sandi C, Venero C, Richter-Levin G (2004) A facilitative role for corticosterone in the acquisition of a spatial task under moderate stress. Learn Mem 11:188-195.

Alfarez DN, Joëls M, Krugers HJ (2003) Chronic unpredictable stress impairs long-term potentiation in rat hippocampal CA1 area and dentate gyrus in vitro. Eur J Neurosci 17:1928-1934.

Arbel I, Kadar T, Silbermann M, Levy A (1994) The effects of long-term corticosterone administration on hippocampal morphology and cognitive performance of middle-aged rats. Brain Res 657:227-235.

Avital A, Segal M, Richter-Levin G (2006) Contrasting roles of corticosteroid receptors in hippocampal plasticity. J Neurosci 26:9130-9134.

Conrad CD, Galea LA, Kuroda Y, McEwen BS (1996) Chronic stress impairs rat spatial memory on the $\mathrm{Y}$ maze, and this effect is blocked by tianeptine pretreatment. Behav Neurosci 110:1321-1334.

Conrad CD, Lupien SJ, Thanasoulis LC, McEwen BS (1997) The effects of type I and type II corticosteroid receptor agonists on exploratory behavior and spatial memory in the Y-maze. Brain Res 759:76-83.

Conrad CD, Lupien SJ, McEwen BS (1999) Support for a bimodal role for type II adrenal steroid receptors in spatial memory. Neurobiol Learn Mem 72:39-46.

Coussens CM, Kerr DS, Abraham WC (1997) Glucocorticoid receptor activation lowers the threshold for NMDA-receptor-dependent homosynaptic long-term depression in the hippocampus through activation of voltage-dependent calcium channels. J Neurophysiol 78:1-9.

Creager R, Dunwiddie T, Lynch G (1980) Paired-pulse and frequency facilitation in the CA1 region of the in vitro rat hippocampus. J Physiol 299:409-424.

de Kloet ER, Oitzl MS, Joëls M (1999) Stress and cognition: are corticosteroids good or bad guys? Trends Neurosci 22:422-426.

DeLuca NA, McCarthy AM, Schaffer PA (1985) Isolation and characterization of deletion mutants of herpes simplex virus type I in the gene incoding immediate-early regulatory protein ICP4. J Virol 56:558-570. de Quervain DJ, Roozendaal B, McGaugh JL (1998) Stress and glucocorticoids impair retrieval of long-term spatial memory. Nature 394:787-790.

Diamond DM, Bennett MC, Fleshner M, Rose GM (1992) Inverted-U relationship between the level of peripheral corticosterone and the magnitude of hippocampal primed burst potentiation. Hippocampus 2:421-430.

Diamond DM, Fleshner M, Ingersoll N, Rose GM (1996) Psychological stress impairs spatial working memory: relevance to electrophysiological studies of hippocampal function. Behav Neurosci 110:661-672.

DiScenna PG, Teyler TJ (1994) Development of inhibitory and excitatory synaptic transmission in the rat dentate gyrus. Hippocampus 4:569-576.

Dudek SM, Bear MF (1993) Bidirectional long-term modification of synaptic effectiveness in the adult and immature hippocampus. J Neurosci 13:2910-2918.

Dumas TC, McLaughlin JR, Ho DY, Lawrence MS, Sapolsky RM (2000) Gene therapies that enhance hippocampal neuron survival after an excitotoxic insult are not equivalent in their ability to maintain synaptic transmission. Exp Neurol 166:180-189.

Dumas TC, Powers EC, Tarapore PE, Sapolsky RM (2004) Overexpression of calbindin $\mathrm{D}(28 \mathrm{k})$ in dentate gyrus granule cells alters mossy fiber presynaptic function and impairs hippocampal-dependent memory. Hippocampus 14:701-709.

Ferguson D, Sapolsky R (2008) Overexpression of mineralocorticoid and transdominant glucocorticoid receptor blocks the impairing effects of glucocorticoids on memory. Hippocampus 18:1103-1111.

Foster TC, McNaughton BL (1991) Long-term enhancement of CA1 synaptic transmission is due to increased quantal size, not quantal content. Hippocampus 1:79-91.

Foy MR, Stanton ME, Levine S, Thompson RF (1987) Behavioral stress impairs long-term potentiation in rodent hippocampus. Behav Neural Biol 48:138-149.

Garcia A, Steiner B, Kronenberg G, Bick-Sander A, Kempermann G (2004) Age-dependent expression of glucocorticoid- and mineralocorticoid receptors on neural precursor cell populations in the adult murine hippocampus. Aging Cell 3:363-371.

Gould E, Woolley CS, Cameron HA, Daniels DC, McEwen BS (1991) Arenal steroids regulate postnatal development of the rat dentate gyrus. II. Effects of glucocorticoids and mineralocorticoids on cell birth. J Comp Neurol 313:486-493.

Han F, Ozawa H, Matsuda K, Nishi M, Kawata M (2005) Colocalization of mineralocorticoid receptor and glucocorticoid receptor in the hippocampus and hypothalamus. Neurosci Res 51:371-381.

Ho DY (1994) Amplicon-based herpes simplex virus vectors. Methods Cell Biol 43:191-210.

Joëls M, de Kloet ER (1990) Mineralocorticoid receptor-mediated changes in membrane properties of rat CAl pyramidal neurons in vitro. Proc Natl Acad Sci U S A 87:4495-4498.

Joëls M, de Kloet ER (1992) Control of neuronal excitability by corticosteroid hormones. Trends Neurosci 15:25-30.

Joëls M, Hesen W, Karst H, de Kloet ER (1994) Steroids and electrical activity in the brain. J Steroid Biochem Mol Biol 49:391-398.

Joëls M, Velzing E, Nair S, Verkuyl JM, Karst H (2003) Acute stress increases calcium current amplitude in rat hippocampus: temporal changes in physiology and gene expression. Eur J Neurosci 18:1315-1324.

Kahle JS, Cotman CW (1993) L-2-Amino-4-phosphonobutanoic acid and $1 S, 3 R$-1-aminocyclopentane-1,3-dicarboxylic acid reduce paired-pulse depression recorded from medial perforant path in the dentate gyrus of rat hippocampal slices. J Pharmacol Exp Ther 266:207-215.

Kaufer D, Ogle WO, Pincus ZS, Clark KL, Nicholas AC, Dinkel KM, Dumas TC, Ferguson D, Lee AL, Winters MA, Sapolsky RM (2004) Restructuring the neuronal stress response with anti-glucocorticoid gene delivery. Nat Neurosci 7:947-953.

Kempermann G (2002) Why new neurons? Possible functions for adult hippocampal neurogenesis. J Neurosci 22:635-638.

Kim JJ, Diamond DM (2002) The stressed hippocampus, synaptic plasticity and lost memories. Nat Rev Neurosci 3:453-462.

Kim JJ, Yoon KS (1998) Stress: metaplastic effects in the hippocampus. Trends Neurosci 21:505-509.

Kim JJ, Foy MR, Thompson RF (1996) Behavioral stress modifies hippocampal plasticity through $N$-methyl-D-aspartate receptor activation. Proc Natl Acad Sci U S A 93:4750-4753.

Kim JJ, Song EY, Kosten TA (2006) Stress effects in the hippocampus: synaptic plasticity and memory. Stress 9:1-11. 
Kolber BJ, Wieczorek L, Muglia LJ (2008) Hypothalamic-pituitary-adrenal axis dysregulation and behavioral analysis of mouse mutants with altered glucocorticoid or mineralocorticoid receptor function. Stress 11:321338.

Li Y, Mu Y, Gage FH (2009) Development of neural circuits in the adult hippocampus. Curr Top Dev Biol 87:149-174.

Liu L, Tsuji M, Takeda H, Takada K, Matsumiya T (1999) Adrenocortical suppression blocks the enhancement of memory storage produced by exposure to psychological stress in rats. Brain Res 821:134-140.

Luine V, Villegas M, Martinez C, McEwen BS (1994) Repeated stress causes reversible impairments of spatial memory performance. Brain Res 639:167-170.

Magariños AM, McEwen BS (1995) Stress-induced atrophy of apical dendrites of hippocampal CA3c neurons: comparison of stressors. Neuroscience 69:83-88.

Malberg JE, Duman RS (2003) Cell proliferation in adult hippocampus is decreased by inescapable stress: reversal by fluoxetine treatment. Neuropsychopharmacology 28:1562-1571.

Martin SJ, Morris RGM (2002) New life in an old idea: the synaptic plasticity and memory hypothesis revisited. Hippocampus 12:609-636.

McEwen BS, De Kloet ER, Rostene W (1986) Adrenal steroid receptors and actions in the nervous system. Physiol Rev 66:1121-1188.

McLaughlin J, Roozendaal B, Dumas T, Gupta A, Ajilore O, Hsieh J, Ho D, Lawrence M, McGaugh JL, Sapolsky R (2000) Sparing of neuronal function postseizure with gene therapy. Proc Natl Acad Sci U S A 97:12804-12809.

Morris RG (1981) Spatial localisation does not depend on the presence of local cues. Learn Motiv 12:239-260.

Nicholas A, Munhoz CD, Ferguson D, Campbell L, Sapolsky R (2006) Enhancing cognition after stress with gene therapy. J Neurosci 26:1163711643 .

Oitzl MS, de Kloet ER (1992) Selective corticosteroid antagonists modulate specific aspects of spatial orientation learning. Behav Neurosci 106:62-71.

Oitzl MS, Fluttert M, de Kloet ER (1994) The effect of corticosterone on reactivity to spatial novelty is mediated by central mineralocorticosteroid receptors. Eur J Neurosci 1:1072-1079.

Pavlides C, Watanabe Y, McEwen B (1993) Effects of glucocorticoids on hippocampal long-term potentiation. Hippocampus 3:183-192.

Pavlides C, Kimura A, Magariños AM, McEwen BS (1994) Type I adrenal steroid receptors prolong hippocampal long-term potentiation. Neuroreport 5:2673-2677.

Pavlides C, Kimura A, Magariños AM, McEwen BS (1995) Hippocampal homosynaptic long-term depression/depotentiation induced by adrenal steroids. Neuroscience 68:379-385.

Pfaff DW, Silva MT, Weiss JM (1971) Telemetered recording of hormone effects on hippocampal neurons. Science 172:394-395.

Pittenger C, Kandel ER (2003) In search of general mechanisms for longlasting plasticity: Aplysia and the hippocampus. Philos Trans R Soc Lond B Biol Sci 358:757-763.

Pugh CR, Tremblay D, Fleshner M, Rudy JW (1997) A selective role for corticosterone in contextual-fear conditioning. Behav Neurosci 111: 503-511.

Reul JM, de Kloet ER (1985) Two receptor systems for corticosterone in rat brain: microdistribution and differential occupation. Endocrinology 117:2505-2511.

Roozendaal B, McGaugh JL (1997) Basolateral amygdala lesions block the memory-enhancing effect of glucocorticoid administration in the dorsal hippocampus of rats. Eur J Neurosci 9:76-83.

Sandi C, Loscertales M, Guaza C (1997) Experience-dependent facilitating effect of corticosterone on spatial memory formation in the water maze. Eur J Neurosci 9:637-642.

Sauro MD, Jorgensen RS, Pedlow CT (2003) Stress, glucocorticoids, and memory: a meta-analytic review. Stress 6:235-245.

Saxe MD, Malleret G, Vronskaya S, Mendez I, Garcia AD, Sofroniew MV, Kandel ER, Hen R (2007) Paradoxical influence of hippocampal neurogenesis on working memory. Proc Natl Acad Sci U S A 104:4642-4646.

Schmidt-Hieber C, Jonas P, Bischofberger J (2004) Enhanced synaptic plasticity in newly generated granule cells of the adult hippocampus. Nature 429:184-187.

Seckl JR (2004) 11beta-hydroxysteroid dehydrogenases: changing glucocorticoid action. Curr Opin Pharmacol 4:597-602.

Shapiro ML, Eichenbaum H (1999) Hippocampus as a memory map: synaptic plasticity and memory encoding by hippocampal neurons. Hippocampus 9:365-384.

Snyder JS, Kee N, Wojtowicz JM (2001) Effects of adult neurogenesis on synaptic plasticity in the rat dentate gyrus. J Neurophysiol 85:2423-2431.

Snyder JS, Hong NS, McDonald RJ, Wojtowicz JM (2005) A role for adult neurogenesis in spatial long-term memory. Neuroscience 130:843-852.

Stein-Behrens B, Mattson MP, Chang I, Yeh M, Sapolsky R (1994) Stress exacerbates neuron loss and cytoskeletal pathology in the hippocampus. J Neurosci 14:5373-5380.

Stienstra CM, Van Der Graaf F, Bosma A, Karten YJ, Hesen W, Joëls M (1998) Synaptic transmission in the rat dentate gyrus after adrenalectomy. Neuroscience 85:1061-1071.

Takahashi T, Kimoto T, Tanabe N, Hattori TA, Yasumatsu N, Kawato S (2002) Corticosterone acutely prolonged $N$-methyl-D-aspartate receptormediated $\mathrm{Ca}^{2+}$ elevation in cultured rat hippocampal neurons. J Neurochem 83:1441-1451.

Tanapat P, Galea LA, Gould E (1998) Stress inhibits the proliferation of granule cell precursors in the developing dentate gyrus. Int J Dev Neurosci 16:235-239.

Vouimba RM, Yaniv D, Richter-Levin G (2007) Glucocorticoid receptors and beta-adrenoceptors in basolateral amygdala modulate synaptic plasticity in hippocampal dentate gyrus, but not in area CA1. Neuropharmacology 52:244-252.

Wagner JJ, Alger BE (1995) GABAergic and developmental influences on homosynaptic LTD and depotentiation in rat hippocampus. J Neurosci 15:1577-1586.

Wang S, Scott BW, Wojtowicz JM (2000) Heterogenous properties of dentate granule neurons in the adult rat. J Neurobiol 42:248-257.

Woolley CS, Gould E, McEwen BS (1990) Exposure to excess glucocorticoids alters dendritic morphology of adult hippocampal pyramidal neurons. Brain Res 531:225-231.

Wright RL, Lightner EN, Harman JS, Meijer OC, Conrad CD (2006) Attenuating corticosterone levels on the day of memory assessment prevents chronic stress-induced impairments in spatial memory. Eur J Neurosci 24:595-605.

Xu L, Anwyl R, Rowan MJ (1997) Behavioural stress facilitates the induction of long-term depression in the hippocampus. Nature 387:497-500.

Yang Y, Zheng X, Wang Y, Cao J, Dong Z, Cai J, Sui N, Xu L (2004) Stress enables synaptic depression in CAl synapses by acute and chronic morphine: possible mechanisms for corticosterone on opiate addiction. J Neurosci 24:2412-2420. 